Platelets are individually encapsulated in monodisperse (CV of $1-4 \%)$ water-in-oil droplets with a mean volume of $14 \mathrm{pL}$ and a diameter of $30 \quad \hat{\mathrm{I}} 1 / 4 \mathrm{~m}$. Droplets are produced with a throughput of $4 \mathrm{kHz}$, with droplets containing a single platelet produced at a rate of $0.25 \mathrm{kHz}$ (following a Poisson distribution). With this method an intrinsic variation in the platelet response to convulxin is observed, that is unrelated to the size of the platelet.

The research entailed the development of a method capable of measuring the intrinsic variation in platelet function. The method will be used to compare the sensitivity profiles of people with known risk factors for arterial thrombosis with the profiles of people with low risk. This presents the possibility to identify a novel prognostic biomarker. Furthermore, a better understanding of the functional heterogeneity of platelets could be used to identify new targets to aid the rational design of new therapeutics. Ideally, this would target only hyperactive platelets, while preserving normal haemostasis.

\section{ATHEROPRONE FLOW ALTERS ATP-INDUCED CALCIUM SIGNALLING IN THE ENDOTHELIUM}

${ }^{1}$ Jack Green*, ${ }^{2}$ Paul Evans, ${ }^{2}$ Heather Wilson. 'University of Sheffield; ${ }^{2}$ Department of Infection, Immunity and Cardiovascular Disease (IICD), University of Sheffield; *Presenting Author

\subsection{6/heartjnl-2016-309890.191}

Introduction The development of the atherosclerotic plaque occurs at distinct points in the vasculature, with areas such as bends and branch points susceptible to plaque formation. The endothelium at these sites is influenced by a disturbed blood flow with low wall shear stress. Numerous studies have shown that stimulation of endothelial cells with this flow pattern induces several inflammatory signalling pathways, but the mechanisms in which this flow pattern is sensed remain unclear.

ATP is released extracellularly from endothelial cells in response to shear stress. The endothelium expresses the ATPgated cation channels P2X4 and P2X7, which have been previously shown to respond to shear stress induced ATP release. Their expression is also increased in the endothelium following inflammatory stimulation. Therefore, we hypothesise that P2X receptors are involved in sensing atheroprone flow and inducing inflammatory signalling, thereby promoting development of atherosclerosis.

Methods Human Umbilical Vein Endothelial Cells (HUVECs) were cultured under flow for $72 \mathrm{~h}$ using an ibidi flow pump system. Atheroprotective flow was applied using $+13 \mathrm{dyn} / \mathrm{cm}^{2}$ and $\pm 4 \mathrm{dyn} / \mathrm{cm}^{2}(0.5 \mathrm{hz})$ was used for atheroprone flow. After flow conditioning, HUVECs were then loaded with a fluorescent calcium dye (CAL-520) and their response to

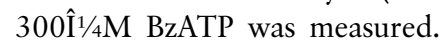

Results Endothelial cells preconditioned with atheroprone flow showed an enhanced calcium response after BzATP stimulation. This enhancement was dependent on extracellular calcium as no increase was detected when performed in calcium free extracellular buffer. The expression level of P2X4 and P2X7 receptors was significantly enhanced in HUVECs preconditioned with atheroprone flow compared to atheroprotective. Moreover the expression of the cell surface ATPase CD39, which breaks down extracellular ATP, was increased under atheroprotective flow. Chemical inhibition of CD39 with ARL-67156 also significantly enhanced the calcium response to BzATP in cells under atheroprotective, but not atheroprone flow.

Conclusions We have identified an enhanced ATP induced calcium response and expression of the ATP-gated P2X4/7 receptors in HUVEC under atheroprone flow conditions. We observed enhanced expression of the cell surface ATPase CD39 in endothelial cells under atheroprotective flow suggesting that extracellular ATP is more rapidly hydrolysed to adenosine under such conditions. We therefore propose that shear stress induced ATP plays a role in specifically activating cells under atheroprone flow and induces inflammatory signalling pathways, underlying atherogenesis susceptibility in vulnerable regions of the vasculature.

\section{HYPOXIA ENHANCES THE REPARATIVE EFFECT OF TISSUE PROTECTIVE ERYTHROPOIETIN AND ITS NON- ERYTHROPOIETIC PEPTIDE ANALOGUE IN AN ENDOTHELIAL CELL INJURY MODEL}

${ }^{1}$ Lamia Heikal ${ }^{*},{ }^{1}$ Pietro Ghezzi, ${ }^{1}$ Manuela Mengozzi, ${ }^{2}$ Martin Feelisch, ${ }^{1}$ Gordon Ferns ${ }^{1}$ University of Sussex; ${ }^{2}$ Faculty of Medicine, University of Southampton; *Presenting Author

\subsection{6/heartjnl-2016-309890.192}

Background Endothelial injury is a critical feature in the early stages of vascular disease. Inflammation and hypoxia are often associated with endothelial injury, stimulating the expression of several cytokines that include erythropoietin (EPO). Endothelial cell-derived EPO appears to be important for protecting the endothelium against ischaemic injury. A non-erythropoietic analogue of EPO; pyroglutamate helix B surface peptide (pHBSP) retains these protective properties of EPO without possessing its erythropoietic effects. The aim of our study was to assess the effects of these molecules in a model of endothelial injury under normoxic and hypoxic conditions.

Method The reparative effects of EPO and pHBSP were assessed under hypoxia $\left(1 \% \mathrm{O}_{2}\right)$ and normoxia $\left(21 \% \mathrm{O}_{2}\right)$ in an in vitro model of endothelial injury (scratch assay). A monolayer of bovine aortic endothelial cells (BAECs), grown to confluence in a multi-well plate, was scratched and the closure of the injured endothelial monolayer was assessed over $24 \mathrm{~h}$. The effects of EPO and pHBSP on BAEC proliferation, chemotaxis and apoptosis were assessed under similar hypoxic conditions in separate experiments. The potential molecular mechanisms of these effects were also explored.

Results Both EPO and pHBSP enhanced scratch closure under hypoxic conditions by $13 \pm 2.6 \%$, and $10 \pm 1.69 \%$ respectively $(\mathrm{p}<0.01)$ compared to normoxic conditions $(3.2 \pm$ $0.9 \%$ and $2.9 \pm 0.3 \%$ for EPO and pHBSP respectively $\mathrm{p}>$ $0.05)$. These effects appeared to be by promoting cell proliferation and migration of BAECs $(\mathrm{p}<0.05)$. EPO also protected BAECs from staurosporine-induced apoptosis under hypoxic conditions. The priming effect of hypoxia was associated with

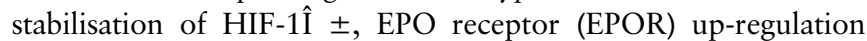
and decreased phosphorylation of endothelial nitric oxide synthase (eNOS) at the Ser-1177 residue. The effect of hypoxia on the latter was rescued by EPO. Hypoxia was associated with a reduction in nitric oxide (NO) production as assessed by its oxidation products nitrite and nitrate, and this was consistent with the oxygen requirement for the endogenous production of NO by NO synthase (NOS). Whilst EPO did not affect NO formation in normoxia, it markedly increased NO production under hypoxic conditions, in a NOS-dependent 